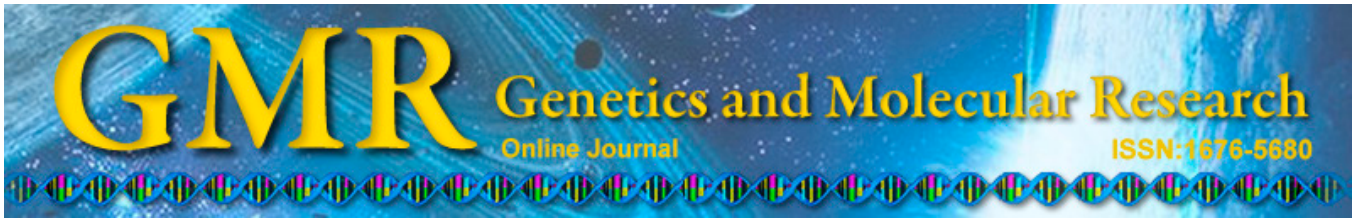

\title{
Associations of single nucleotide polymorphisms in the Pygo 2 coding sequence with idiopathic oligospermia and azoospermia
}

S.-Q. Ge $\mathrm{e}^{1,2,5,6,7}$,J. Grifin ${ }^{2}$, L.-H. Liu ${ }^{2}$ K.I. Aston ${ }^{2}$ L. Simon ${ }^{2}$ T.G. Jenkins ${ }^{2}$ B.R. Emery ${ }^{2}$ and D.T. Carrell ${ }^{2,3,4}$

${ }^{1}$ Hebei University Health Science Center, Baoding, China

${ }^{2}$ Andrology and IVF Laboratories, Division of Urology,

Department of Surgery, University of Utah School of Medicine,

Salt Lake City, UT, USA

${ }^{3}$ Department of Obstetrics and Gynecology,

University of Utah School of Medicine, Salt Lake City, UT, USA

${ }^{4}$ Department of Physiology, University of Utah School of Medicine,

Salt Lake City, UT, USA

${ }^{5}$ Institute for Reproductive Medicine of Hebei University, Baoding, China

${ }^{6}$ The Center for Reproductive Medicine of Affiliated Hospital of Hebei

University, Baoding, China

${ }^{7}$ Hebei Research Institute for Family Planning, Shijiazhuang, China

Corresponding authors: S.-Q. Ge / D.T. Carrell

E-mail: gesq67@163.com / douglas.carrell@hsc.utah.edu

Genet. Mol. Res. 14 (3): 9053-9061 (2015)

Received January 30, 2015

Accepted May 24, 2015

Published August 7, 2015

DOI http://dx.doi.org/10.4238/2015.August.7.14

ABSTRACT. Male infertility is often associated with a decreased sperm count. The Pygo 2 gene is expressed in the elongating spermatid during chromatin remodeling; thus impairment in PYGO2 function might lead to spermatogenic arrest, sperm count reduction, and subsequent infertility. The aim of this study was to identify mutations in Pygo2 that might lead to idiopathic oligospermia and azoospermia. DNA was isolated from venous blood from 77 men with normal fertility and 195 
men with idiopathic oligospermia or azoospermia. Polymerase chain reaction-sequencing analysis was performed for the three Pygo 2 coding regions. Non-synonymous single nucleotide polymorphisms (SNPs) were detected and analyzed using SIFT, Polyphen-2, and Mutation Taster softwares to identify possible changes in protein structure that could affect phenotype. Pygo2 sequencing was successful for 178 patients (30 with mild or moderate oligospermia, 57 with severe oligospermia, and 91 with azoospermia). Three previously reported nonsynonymous SNPs were identified in patients with azoospermia or severe oligospermic but not in those with mild or moderate oligozoopermia or normozoospermia. SNPs rs61758740 (M141I) and rs141722381 (N240I) cause the replacement of one hydrophobic or hydrophilic amino acid, respectively, with another, and SNP rs61758741 (K261E) causes the replacement of a basic amino acid with an acidic one. The software predictions demonstrated that SNP rs141722381 would likely result in disrupted tertiary protein structure and thus could be involved in disease pathogenesis. Overall, this study demonstrated that SNPs in the coding region of Pygo2 might be one of the causative factors in idiopathic oligospermia and azoospermia, resulting in male infertility.

Key words: Idiopathic oligospermia; Idiopathic azoospermia; SNPs; Pygo2 gene

\section{INTRODUCTION}

The past few decades have seen a reduction in sperm counts and a proportionate increase in male infertility (Carlsen et al., 1992). Microdeletion of the Y chromosome, cystic fibrosis, varicocele, Klinefelter syndrome, and chemotherapy or radiotherapy are among the few special circumstances, which are known to lead to a reduction in sperm count. On the other hand, idiopathic oligozoospermia and azoospermia, which lead to infertility, are multifactorial diseases whose etiologies are not well understood. Thus, many researchers are committed to find the underlying causes of these disorders.

Pygopus (PYGO) is a member of the evolutionarily conserved C-terminal plant homology domain (PHD)-finger protein family that has two mammalian homologues, Pygo1 and 2. The Pygo2 gene has a total of three exons: ENSE 00001447170, located between nucleotide positions 2106-2380 and encoding the amino acids Met1-Gly35; ENSE00001619898, located between nt 2828-2877 and encoding Gly35-Gln51; and ENSE00001073617, located between nt 4008-6828 and encoding Gly52-Gly406. The human PYGO2 protein is localized in the nucleus. It has two domains: the PHD zinc finger motif, and the N-terminal homology domain (NHD). As a co-activator of the Wnt pathway through its PHD domain, PYGO2, together with the BCL9, $\beta$-catenin, and LEF/TCF transcription factors constitutes a transcription complex that activate the expression of specific target genes. The NHD domain plays an important role in transcriptional activation by participating in the recruitment of histone modification factors, and is also involved in histone methylation (Gu et al., 2009). In the process of sperm formation (spermiogenesis), the Pygo2 gene is expressed during remodeling of the elongated sperm cell chromatin. Research on the effects of Pygo2 mutations in mice has shown that limited Pygo2 
function during sperm formation would lead to a selective reduction in protamine $(P 1, P 2)$, transitional protein 2 (Tnp2), and H1fnt gene expression after meiosis reduction. In addition, modifications of the highly acetylated histone H3 changed dramatically (Nair et al., 2008), which would likely result in sperm count reduction or in stagnation leading to infertility.

In order to detect whether mutation of the Pygo2 gene causes idiopathic oligozoospermia or azoospermia in humans, the Pygo2 gene protein coding sequences from 178 men with idiopathic oligozoospermia and azoospermia (representing the number of successful sequence analyses from an original cohort of 195 patients) and 77 men with normal fertility were compared. We confirmed the existence of single nucleotide polymorphism (SNP) mutations in the Pygo2 gene in patients with oligozoospermia and azoospermia. Software analysis of the SNP mutations was used to explore the possibility of variant PYGO2 protein-induced (M141>I141; N240>I240; K261>E261) structural damage and concomitant, illuminating the potential relevance association of SNPs in the Pygo2 coding regions with idiopathic oligospermia and azoospermia.

\section{MATERIAL AND METHODS}

\section{Subjects}

This research was approved by the Ethics System Review Committee of the University of Utah. We enrolled 77 men with normal fertility as well as 195 men with idiopathic oligozoospermia and azoospermia in this study. According to the World Health Organization (WHO) semen clinical classification, patients were divided into three groups: mild or moderate oligozoospermia $\left(5-20 \times 10^{6} \mathrm{sperm} / \mathrm{mL} ; 31 \mathrm{men}\right)$, severe oligozoospermia $(0-5 \times 106$ $\mathrm{sperm} / \mathrm{mL} ; 63 \mathrm{men})$, and azoospermia $\left(0 \times 10^{6} \mathrm{sperm} / \mathrm{mL} ; 101 \mathrm{men}\right)$. All patients were seen by the Andrology and IVF Laboratories at the University of Utah. Patient ages were between 25 and 50 years. Men with Y-chromosome microdeletions, cystic fibrosis, varicocele, Klinefelter syndrome, a history of chemotherapy or radiotherapy, orchitis, cryptorchidism, inguinal hernia, or other special circumstances were excluded.

\section{Methods}

\section{DNA isolation}

DNA was extracted from venous blood collected from patients, using a DNA extraction kit (Minneapolis, MN, USA). The final DNA concentration was normalized to $20 \mathrm{ng} / \mu \mathrm{L}$ before storage at $-80^{\circ} \mathrm{C}$.

\section{Polymerase chain reaction (PCR) amplification}

The PCR was carried out in a system of $23 \mu \mathrm{L}$, containing $0.02 \mu \mathrm{g}$ genomic DNA. Amplification conditions were as follows: pre-denaturation at $95^{\circ} \mathrm{C}$ for $5 \mathrm{~min}$; denaturation at $95^{\circ} \mathrm{C}$ for $30 \mathrm{~s}$, annealing at the optimum temperature for each primer (Table 1) for $30 \mathrm{~s}$, and extension at $72^{\circ} \mathrm{C}$ for $1 \mathrm{~min}$ for a total of 35 cycles, with a final extension at $72^{\circ} \mathrm{C}$ for $7 \mathrm{~min}$. Because of the long length of the third protein coding sequence in the Pygo2 gene, this exon was divided into three mutually intersecting portions (3-1,3-2, and 3-3) for primer design and PCR amplification. 
Table 1. PCR amplification primers for the Pygo2 gene coding regions.

\begin{tabular}{|c|c|c|c|c|}
\hline $\begin{array}{l}\text { Protein coding } \\
\text { sequence number }\end{array}$ & Forward primer $\left(5^{\prime}-3^{\prime}\right)$ & Reverse primer $\left(5^{\prime}-3^{\prime}\right)$ & $\begin{array}{c}\text { Annealing } \\
\text { temperature }\left({ }^{\circ} \mathrm{C}\right)\end{array}$ & $\begin{array}{l}\text { Product } \\
\text { size (bp) }\end{array}$ \\
\hline 1 & CTCGGCGCTTCCCTGTCGTC & GCCCCCAACCCCTGGAGGAT & 64 & 400 \\
\hline 2 & CCTCAGGGAAACTTGTCTGC & AACCAGGACAGGAAACATGG & 60 & 351 \\
\hline $3-1$ & TGAGCTGCCCACAGTATCAG & GAGAGGAGAAGGGCCAAAAG & 60 & 640 \\
\hline $3-2$ & ACAGCCTCCCAGAGCAGAG & ATGCCCTCACGGATGTAGAC & 60 & 594 \\
\hline $3-3$ & GGCTGCTGACCACTGAAGC & TTGGAGGAAGCAAAGGAGTA & 60 & 675 \\
\hline
\end{tabular}

$\mathrm{PCR}=$ polymerase chain reaction.

\section{Gene sequencing and SNP detection}

PCR products were treated with ExoSAP-IT (Minneapolis, MN, USA) (a mixture of exonuclease and shrimp alkaline phosphatase) to remove residual primers and dNTPs. Gene sequencing was performed by the Experimental Center of Utah University (Salt Lake City, UT, USA). Sequence trace analysis and SNP detection were performed using the Phred software package (www.phrap.org).

\section{SNP mutation analysis}

SNP mutations in Pygo2 identified by sequencing were analyzed using SIFT (Kumar et al., 2009), Polyphen-2 (Adzhubei et al., 2010) and Mutation Taster (Schwarz et al., 2010) algorithms to explore the likelihood of associated disruption of the PYGO2 protein structure and concomitant disease-induction. The three Pygo2 protein-coding sequences were compared among patients with idiopathic oligozoospermia and azoospermia and with normal males to explore the potential relevance of gene mutation to male infertility.

\section{RESULTS}

Pygo2 gene sequencing was successful for 178 of the original 195 patients (30/31 cases of mild or moderate oligozoospermia, 57/63 cases of severe oligozoospermia, and 91/101 cases of azoospermia). The non-synonymous SNP mutations rs61758740 and rs141722381 were identified at two separate sites in three patients with azoospermia, and the non-synonymous SNP mutations rs61758741 was identified in one patient with severe oligospermia. No SNPs were found in patients with mild or moderate oligozoopermia or normozoospermia. These three mutations had been previously reported in the National Center for Biotechnology Information SNP database ((NCBI SNPdb) (Table 2).

\begin{tabular}{|c|c|c|c|c|c|c|}
\hline Disorder $(\mathrm{N})$ & SNP & Nucleotide substitution & $\begin{array}{l}\text { Nonsynonymous } \\
\text { mutation }\end{array}$ & SIFT & Polyphen-2 & Mutation Taster \\
\hline Azoospermia (2) & rs61758740 & G2206>A (ATG-ATA) & M141I & No effect on protein structure & No effect on protein structure & Non-disease causing \\
\hline Azoospermia (1) & rs141722381 & A2502 > T (AAC-ATC) & $\mathrm{N} 240 \mathrm{I}$ & Disrupts protein structure & Disrupts protein structure & Disease causing \\
\hline Severe oligospermia (1) & rs61758741 & A2564>G (AAG-GAG) & $\mathrm{K} 261 \mathrm{E}$ & No effect on protein structure & No effect on protein structure & Non-disease causing \\
\hline
\end{tabular}


As shown in Table 2, among the three SNPs identified in the Pygo2 gene, rs61758740 is located at nucleotide position 2206 of the Pygo2 gene, and leads to the replacement of a methionine $(\mathrm{M}$; isoelectric point $(\mathrm{pI})=5.74)$ with an isoleucine $(\mathrm{I} ; \mathrm{pI}=6.02)$ residue: M141I; rs61758741 is located at nt 2564 and results in the replacement of a lysine $(\mathrm{K} ; \mathrm{pI}=9.74)$ with a glutamic acid $(\mathrm{E} ; \mathrm{pI}=3.27)$ residue: K261E; and rs 141722381, located at nt 2502, leads to the replacement of an asparagine $(\mathrm{N} ; \mathrm{pI}=5.41)$ with an isoleucine $(\mathrm{I} ; \mathrm{pI}=6.02)$ residue: N240I. These three SNP mutations in patients with azoospermia and oligospermia therefore alter three of the amino acids in the PYGO2 protein, all of which are located between the Nterminal NHD and C-terminal PHD domains. A schematic illustrating the corresponding sites of mutation is shown in Figure 1.

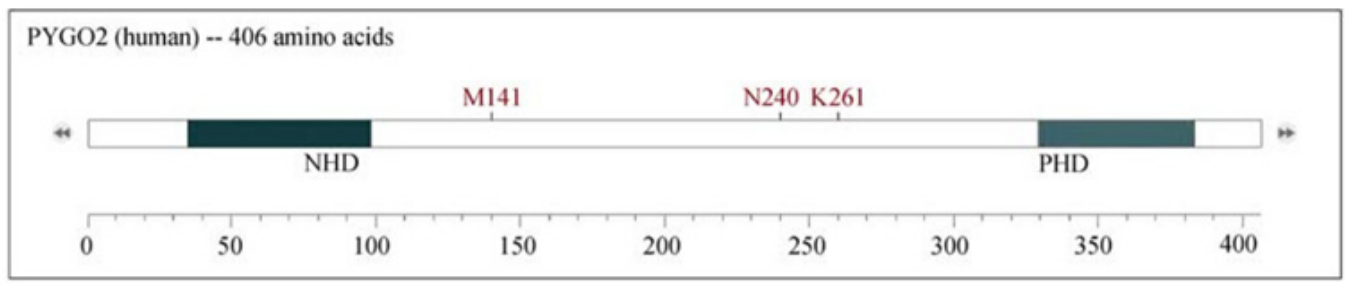

Figure 1. Schematic of the PYGO2 protein amino acid sites corresponding to the three oligospermia and azoospermia SNP mutations in relation to the NHD and PHD structural domains. SNP $=$ single nucleotide polymorphism; NHD = N-terminal homology domain; $\mathrm{PHD}=\mathrm{C}$-terminal plant homology domain.

The potential functional effects of the three non-synonymous SNP mutations were analyzed by SIFT, Polyphen- 2, and Mutation Taster software algorithms. The SIFT and Polyphen-2 values for rs61758740 were 0.24 and 0.005 , respectively, which were consistent with the negative results predicted by Mutation Taster, indicating that the mutation was unlikely to result in changes to the protein structure that might lead to disease. Similarly, the SIFT and Polyphen-2 values obtained for rs61758741 were 1 and 0.435 , respectively, and the diseaserelated results predicted by Mutation Taster were also negative. However, the SIFT and Polyphen-2 analysis values for rs 141722381 were 0.05 and 0.968 , indicating that this mutation might lead to disruption of the PYGO2 protein structure, and the disease-related results predicted by Mutation Taster were positive as well. Therefore, following analysis by three separate software algorithms, the rs141722381 mutation identified in patients with azoospermia was considered to be a likely cause of PYGO2 protein structural damage, and also showed a positive correlation with disease-induction.

\section{DISCUSSION}

Spermatogenesis includes the following steps: primordial germ cells first develop into spermatogonia, and then develop into spermatocytes; spermatocytes become round germ cells after two meiotic divisions; and round sperm cells become mature sperm after metamorphosis. In this complex cellular differentiation process, all cellular events are carried out with the temporal regulation of multiple genes (Ge et al., 2008; Matzuk and Burns, 2012). Disturbances in any factors related to the expression of spermatogenesis genes might therefore ultimately lead to reductions in sperm quality and number. 
Idiopathic oligozoospermia and azoospermia is a multi-factor disorder, which has been well studied by multiple laboratories. Over the past several years, A et al. (2006) and $\mathrm{Li}$ et al. (2007) have demonstrated that microdeletion of chromosome Y represents an important mechanism underlying certain cases of idiopathic azoospermia and oligozoospermia. Routine screening for chromosome Y microdeletions in patients with idiopathic azoospermia and oligozoospermia is therefore necessary, with cytogenetic analysis proving to be very helpful in the accurate diagnosis of the disease (Chang et al., 2012). A et al. (2006) have suggested that deletion of all copies of the $D A Z$ gene is the common genetic cause for patients with severe oligozoospermia, and that $D A Z 1 / D A Z 2$ deletion is a high-risk factor for development of these disorders. Qi et al. (2011) confirmed that the abnormal chromosomal regions in male patients with infertility can be detected by quantitative fluorescent PCR (QF-PCR) technologies quickly, directly and reliably, and that this technique permits discovery of abnormalities in fine chromosomal structure at an early stage, yielding results consistent with full karyotype analysis and with $A Z F$ microdeletion results by PCR. Zhang et al. (2006) considered that abnormalities of the synaptonemal complex and its encoding genes could cause spermatogenetic malfunction in an infertile mouse; in support of this supposition, the murine azoospermia caused by single base deletion in a synaptonemal complex coding gene has since been confirmed by findings in human patients with primary infertility. Similarly, by studying the relationship between glutathione S-transferase T1 (GSTT1) gene polymorphisms and idiopathic azoospermia and oligozoospermia, Wu et al. (2007) found that GSTT1 was related to idiopathic azoospermia and oligozoospermia; furthermore, the GSTT1 deletion genotype was also shown to be a risk factor for idiopathic azoospermia and oligozoospermia.

Of the many genes related to spermatogenesis, Gu et al. (2009) reported that mutation in Pygo 2 could reduce gene expression after meiosis, leading to the decreased production of PRM1, PRM2, TNP2, and H1fnt, which are correlated with chromatin remodeling in male germ cells leading to nuclear enrichment. In this study, sperm cells of Pygo2 mutant mice were shown to have lost the capacity for acetylation of histone $\mathrm{H} 3$ at its 9th (H3K9) and 14th (H3K14) sites during the ninth stage of spermatogenesis, indicating that PYGO2 might activate gene expression during the spermiogenesis process by promoting acetylation of these two histone residues. The PHD motif of PYGO2 might bind to histone H3 tails trimethylated on lysine 4 (H3K4me3), which are associated with the activation of gene transcription; whereas the NHD might play an important role in transcriptional activation through the recruitment of histone modification factors (Li et al., 2006; Peña et al., 2006; Shi et al., 2006). Phenotypically, the knockout of Pygo2 caused severe dysplasia, including lens hypoplasia and kidney malformation, eventually leading to the death of the embryo (Li et al., 2007; Schwab et al., 2007; Song et al., 2007). Overall, therefore, the process of transcriptional activation of PYGO2 target genes was proposed to be: binding of the PYGO2 transcription complex to select $\mathrm{H} 3 \mathrm{~K} 4 \mathrm{me} 3$ modified genes, with concomitant promotion of additional histone modification (H3K9 and H3K4 acetylation), leading to transcriptional activation (Zhang et al., 2011).

Numerous studies have shown that rare SNPs were closely associated with asthma, diabetes, hypertension, spontaneous autoimmune disease, Parkinson's syndrome, autism, cancer, and other complex human diseases (Han et al., 2011; Lehne et al., 2011; Li et al., 2012a,b). Therefore, we examined whether SNP mutations in the Pygo2 gene caused idiopathic oligozoospermia and azoospermia. We identified two kinds of non-synonymous SNP mutations in the Pygo 2 coding genes of patients with azoospermia, and one kind of non-synonymous SNP mutations in patients with severe oligozoospermia (Table 2). 
Mutation at the rs61758740 site caused the 141st methionine of the PYGO2 protein to be changed to isoleucine; the presence of this conservative change might be attributed to the fact that isoleucine and methionine are both essential amino acids with a hydrophobic side chain, with similar pIs ( $\mathrm{pIM}=5.74$; $\mathrm{pII}=6.02)$; the correlation between the amino acid change, disruption of protein structure, and disease induction were found to be negative by three separate software algorithms. The rs61758741 SNP resulted in the mutation of lysine to glutamate at the 261 st residue of the PYGO2 protein. Theoretically, replacement of the basic amino acid lysine ( $\mathrm{pIK}=9.74)$ at this position in the PYGO2 protein with the acidic amino acid glutamate ( $\mathrm{pIE}=3.27$ ) should influence protein folding, which might lead to disruption of its tertiary structure and a change in function; however, the results following analysis by three separate software algorithms were all negative.

rs141722381 variation led to the mutation of an asparagine into an isoleucine at the 240th residue of the PYGO2 protein, located between the NHD and PHD structural domains. Following the change of an asparagine ( $\mathrm{pIN}=5.41)$ residue, with hydrophilic side chains, to an isoleucine $(\mathrm{pII}=6.02)$ residue with hydrophobic side chains, the lack of the free carboxyl and amino groups of the original asparagine residue might be expected to cause disruption of the PYGO2 tertiary structure, thereby affecting its function. The three software analyses also found that the non-synonymous SNP mutation at this site was positively correlated with tertiary structure disruption of the PYGO2 protein, which might induce disease.

The rs 141722381 mutation reported in the SNP database was originally detected through the 1000 Genomes Project of the European Bioinformatics Institute (EBI) and NCBI. Therein, the $\mathrm{A}>\mathrm{T}$ mutation rate was only $0.1 \%$; however, male infertility was also included among the random 1000 ordinary subjects sampled (1000 Genomes Project Consortium et al., 2010). Therefore, the reported rs141722381 allele frequencies in the database do not negate that potential of Pygo2 as the pathogenic gene. In this study, SNPs were not detected in the Pygo2 genes of 77 men with normal fertility, whereas one instance of rs141722381 mutation was found in the group of 91 patients with azoospermia. Combined with the positive results from the software analysis, this detection rate demonstrated that SNPs in the Pygo2 gene might be predisposing factors for oligozoospermia and azoospermia.

Disruption of the tertiary structure of the PYGO2 protein would likely cause a series of changes in domain functionality, which might result in the inability of PYGO2 to bind to the BCL9, $\beta$-catenin, and LEF/TCF transcription factors, as well as to the apparent modifying factor H3K4me3. This deficit might subsequently negatively influence the transcription of target genes in the Wnt pathway (Gu et al., 2009), resulting in decreased spermatogenesis, reduction in sperm count, or complete lack of sperm.

Our laboratory has achieved previous success in the detection of mutations in a single gene (CREM) for male infertility (Christensen et al., 2006). CREM is a key transcription factor for prototype spermatid differentiation into mature sperm. Different haplotypes of the CREM-activating factor in testicular tissues could cause low male fertility. Here we have identified point mutations in the Pygo2 gene in patients with oligozoospermia and azoospermia, indicating that the etiology of a multi-factor disease might be ascertained at least in part by detection of a single gene mutation. Although this method is relatively labor intensive and time consuming, it might prove to be a repeatable and reliable way to study rare mutations.

Gene copy number variants (CNVs) have also been considered to be associated with human oligozoospermia (Tüttelmann et al., 2011). Furthermore, other studies have shown that certain Y-chromosome haplogroup changes were associated with the reduction of sperm 
count (Kuroki et al., 1999; Krausz et al., 2001; Yang et al., 2008). Epigenetic studies of sperm have also shown that protamination, histone modification, DNA methylation, and non-coding RNAs all are factors that regulate gene expression, which plays an important role in regulating spermatogenesis (Carrell, 2012). Therefore, dysfunction of any epigenetic modification factors will likely affect spermatogenesis, resulting in a reduction in fertilization, embryo implantation, and pregnancy rates (Nanassy and Carrell, 2008).

In summary, idiopathic oligozoospermia and azoospermia is a type of multi-factor disease, and SNPs in the Pygo2 coding gene might be one of the predisposing factors. A comprehensive understanding of the etiologies of this disorder requires further and in-depth studies using a large research sample and the combination of multiple gene-specific screens, $\mathrm{CNV}$ analysis, Y-chromosome haplogroup analysis, and the detection of epigenetic modifying factors, in addition to SNP variance analysis.

\section{Conflicts of interest}

The authors declare no conflict of interest.

\section{ACKNOWLEDGMENTS}

Research supported by the University of Utah Andrology Laboratory Project, the Hebei Province Natural Science Foundation of China (\#H2013201259), the Hebei 2013

Returned Overseas Research Activities (\#C2013005002), and the National 2014 Returned Overseas Research Activities (\#7028505101).

\section{REFERENCES}

A ZC, Yang Y, Zhang SZ and Lin L (2006). Study on DAZ gene copy deletion in severe oligozoospermia sperm donor for ICSI. Yi Chuan 28: 1057-1060.

Adzhubei IA, Schmidt S, Peshkin L, Ramensky VE, et al. (2010). A method and server for predicting damaging missense mutations. Nat. Methods 7: 248-249.

Carlsen E, Giwercman A, Keiding N and Skakkebaek N (1992). Evidence for decreasing quality of semen during past 50 years. BMJ 305: 609-613.

Carrell DT (2012). Epigenetics of the male gamete. Fertil. Steril. 97: 267-274.

Chang L, Gao XF, Yang LP, Jiao LP, et al. (2012). 609 cases patients with no less sperm and the sperm cytogenetic analysis. China Healthy Birth Genet. J. 18: 40-42.

Christensen GL, Wooding SP, Ivanov IP, Atkins JF, et al. (2006). Sequencing and haplotype analysis of the activator of CREM in the testis (ACT) gene in populations of fertile and infertile males. Mol. Hum. Reprod. 12: 257-262.

Ge SQ, Kang XJ, Liu GR and Mu SM (2008). Genes involved in spermatogenesis. Yi Chuan 30: 3-12.

Gu B, Sun P, Yuan Y, Moraes RC, et al. (2009). Pygo2 expands mammary progenitor cells by facilitating histone H3 K4 methylation. J. Cell Biol. 185: 811-826.

Han B, Chen XW and Talebizadeh Z (2011). FEPI-MB: identifying SNPs - disease association using a Markov Blanketbased approach. BMC Bioinformatics 12: S3.

Krausz C, Quintana-Murci L, Rajpert-De Meyts E, Jørgensen N, et al. (2001). Identification of a Y chromosome haplogroup associated with reduced sperm counts. Hum. Mol. Genet. 10: 1873-1877.

Kumar P, Henikoff S and Ng PC (2009). Predicting the effects of coding non-synonymous variants on protein function using the SIFT algorithm. Nat. Protoc. 4: 1073-1081.

Kuroki Y, Iwamoto T, Lee J, Yoshiike M, et al. (1999). Spermatogenic ability is different among males in different Y chromosome lineage. J. Hum. Genet. 44: 289-292.

Lehne B, Lewis CM, Schlitt T and Khanin R (2011). From SNPs to genes: disease association at the gene level. PLoS 
One 6: e20133

Li BA, Rhéaume C, Teng A, Bilanchone V, et al. (2007). Developmental phenotypes and reduced Wnt signaling in mice deficient for pygopus 2. Genesis 45: 318-325.

Li H, Ilin S, Wang W, Duncan EM, et al. (2006). Molecular basis for site-specific read-out of histone H3K4me3 by the BPTF PHD finger of NURF. Nature 442: 91-95.

Li H, Lee Y, Chen JL, Rebman E, et al. (2012a). Complex-disease networks of trait-associated single-nucleotide polymorphisms (SNPs) unveiled by information theory. J. Am. Med. Inform. Assoc. 19: 295-305.

Li K, Tang BS, Yu RL, Lv ZY, et al. (2012b). Association study between two novel single nucleotide polymorphisms and sporadic Parkinson's disease in Chinese Han population. Neurosci. Lett. 517: 56-59.

Matzuk MM and Burns KH (2012). Genetics of mammalian reproduction: modeling the end of the germline. Annu. Rev. Physiol. 74: 503-528.

Nair M, Nagamori I, Sun P, Mishra DP, et al. (2008). Nuclear regulator PYGO2 controls spermiogenesis and histone H3 acetylation. Dev. Biol. 320: 446-455.

Nanassy L and Carrell DT (2008). Paternal effects on early embryogenesis. J. Exp. Clin. Assist. Reprod. 5: 2.

Peña PV, Davrazou F, Shi X, Walter KL, et al. (2006). Molecular mechanism of histone H3K4me3 recognition by plant homeodomain of ING2. Nature 442: 100-103.

Qi ML, Zhang YY, Liu XL, He R, et al. (2011). Chromosomal abnormality diagnosis of male infertility by QF-PCR. $Y i$ Chuan 33: 895-900.

Schwab KR, Patterson LT, Hartman HA, Song N, et al. (2007). Pygo1 and Pygo2 roles in Wnt signaling in mammalian kidney development. BMC Biol. 5: 15.

Schwarz JM, Rödelsperger C, Schuelke M and Seelow D (2010). MutationTaster evaluates disease-causing potential of sequence alterations. Nat. Methods 7: 575-576.

Shi X, Hong T, Walter KL, Ewalt M, et al. (2006). ING2 PHD domain links histone H3 lysine 4 methylation to active gene repression. Nature 442: 96-99.

Song N, Schwab KR, Patterson LT, Yamaguchi T, et al. (2007). Pygopus 2 has a crucial, Wnt pathway-independent function in lens induction. Development 134: 1873-1885.

Tüttelmann F, Simoni M, Kliesch S, Ledig S, et al. (2011). Copy number variants in patients with severe oligozoospermia and Sertoli-cell-only syndrome. PLoS One 6: e19426.

Wu QF, Xing JP, Sun JH, Xue W, et al. (2007). Genetic polymorphism of glutathione S-transferase T1 associated with idiopathic azoospermia and oligospermia. Natl. J. Androl. 13: 407-410.

Yang Y, Ma M, Li L, Zhang W, et al. (2008). Evidence for the association of Y-chromosome haplogroups with susceptibility to spermatogenic failure in a Chinese Han population. J. Med. Genet. 45: 210-215.

Zhang W, Zhang SZ and A ZC (2006). Synaptonemal complex - an essential role in etiology of idiopathic azoospermia. Yi Chuan 28: 231-235.

Zhang XJ, Liu ML and Jia MC (2011). Regulation of gene expression during spermatogenesis at transcriptional level. $Y i$ Chuan 33: 1300-1307.

Zhou-Cun A, Yang Y, Zhang SZ, Zhang W, et al. (2006). Chromosomal abnormality and Y chromosome microdeletion in Chinese patients with azoospermia or severe oligozoospermia. Yi ChuanXue Bao 33: 111-116.

1000 Genomes Project Consortium, Abecasis GR, Altschuler D, Auton A, et al. (2010). A map of human genome variation from population-scale sequencing. Nature 467: 1061-1073. 